\title{
I CONCURSO NACIONAL DE DIBUJO Y PINTURA: "UNA MIRADA CRÍTICA AL PASADO DE LA ESCUELA"
}

Pablo Álvarez Domínguez Facultad de Ciencias de la Educación, Universidad de Sevilla pabloalvarez@us.es

Recientemente, se entendió que una de las mejores formas para divulgar a nivel internacional el trabajo de difusión del patrimonio educativo que se hace desde el Museo Pedagógico de la Facultad de Ciencias de la Educación de la Universidad de Sevilla, así como desde otros Museos Pedagógicos de España, tenía que ver con la organización de un Concurso Nacional de Dibujo y Pintura, que en su primera edición tuviera como lema marco, el de "Una mirada crítica al pasado de la Escuela". Para la organización del Concurso, se contó con el apoyo y colaboración de la Sociedad Española para el Estudio del Patrimonio Histórico Educativo (SEPHE), del Vicerrectorado de Investigación de la Universidad de Sevilla y de la Facultad de Ciencias de la Educación de la misma Universidad.

El I Concurso Nacional de Dibujo y Pintura: "Una mirada crítica al pasado de la Escuela", se planteó con el objetivo de propiciar entre profesionales y aficionados ligados al mundo del dibujo y la pintura, una mirada artista y crítica al pasado de la escuela como espacio cultural, socializador y transmisor de valores. La temática del concurso tuvo que ver con el patrimonio educativo y/o ajuar etnográfico de la escuela que se conserva en el presente.

Bases del concurso:

http://comunicacion.us.es/sites/default/files/bases_i_concurso_nacional_dibujo_y_pintura._m useo_pedagogico_us_1.pdf

El pasado día 22 de noviembre de 2016, se resolvía este concurso, con tres premios y un accésit, que se concretó en los siguientes términos: 


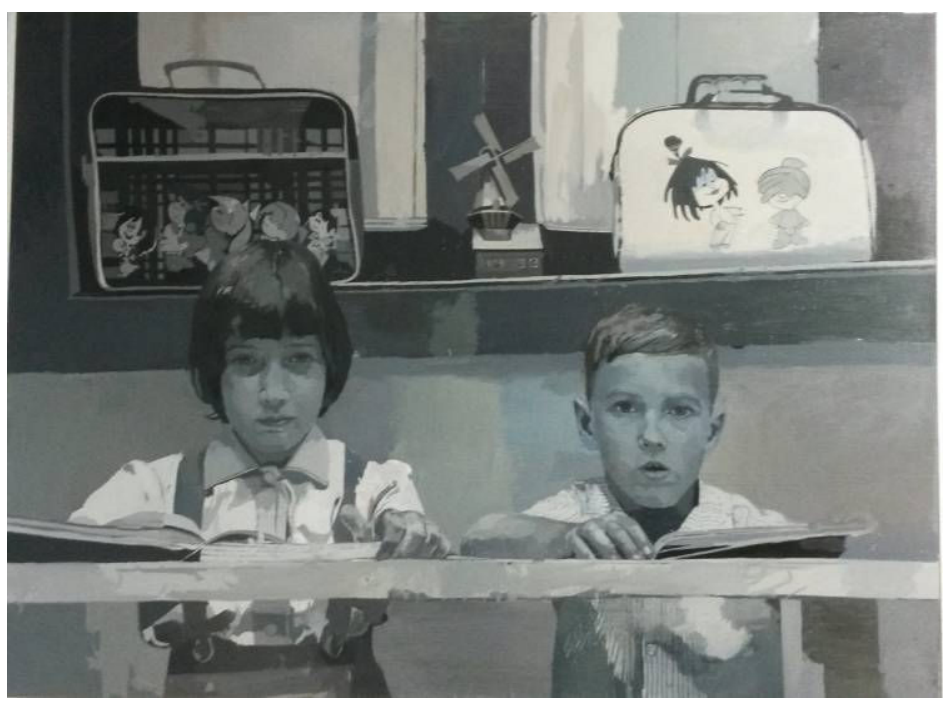

PRIMER PREMIO

D. Gonzalo Rodríguez Gómez

"La instantánea de una generación"

(Óleo sobre tela. $97 \times 130 \mathrm{~cm}$ )

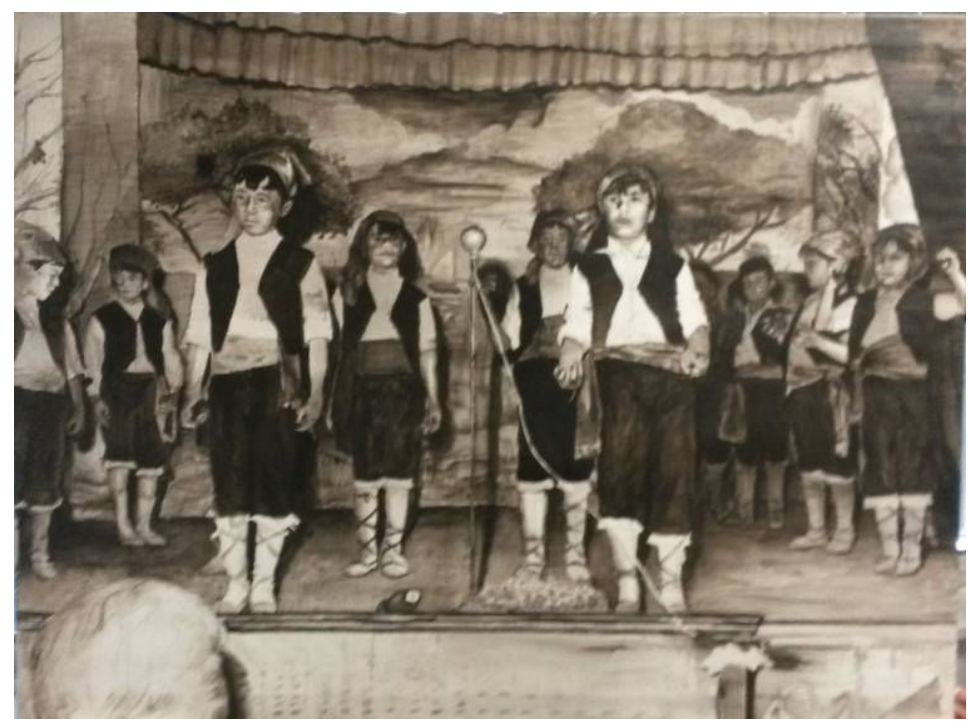

SEGUNDO PREMIO

D. Alberto Crespo Albenca

"El baile"

(Acrílico sobre tela. 97x130 cm) 


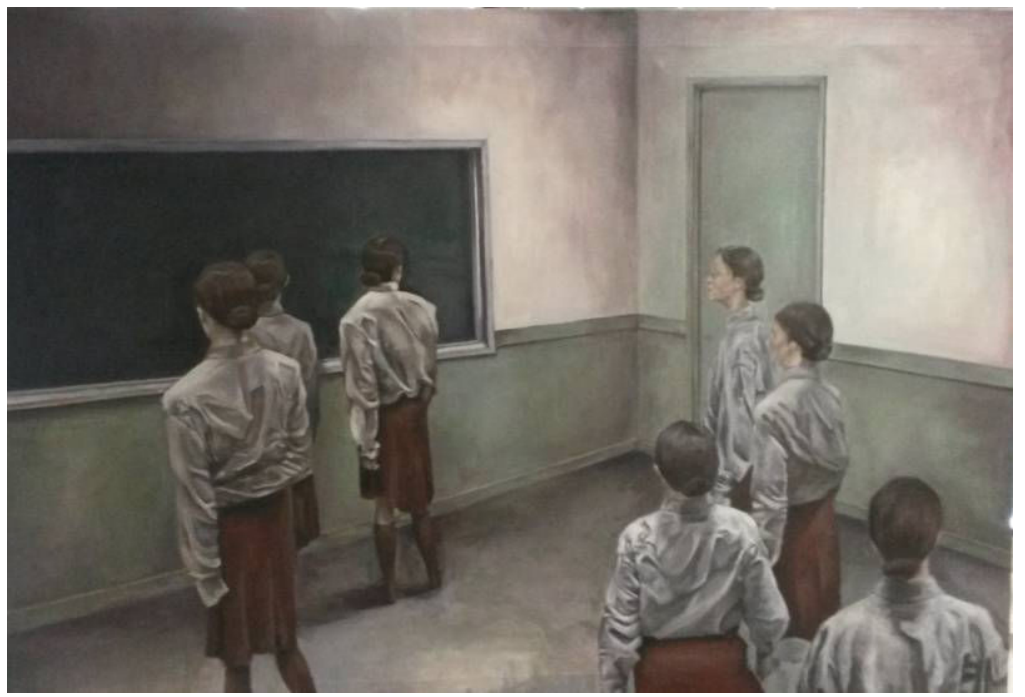

TERCER PREMIO

$\mathrm{D}^{\mathrm{a}}$. Marina Iglesias Mateos

"Cámara"

(Óleo sobre lienzo. 90x130 cm)

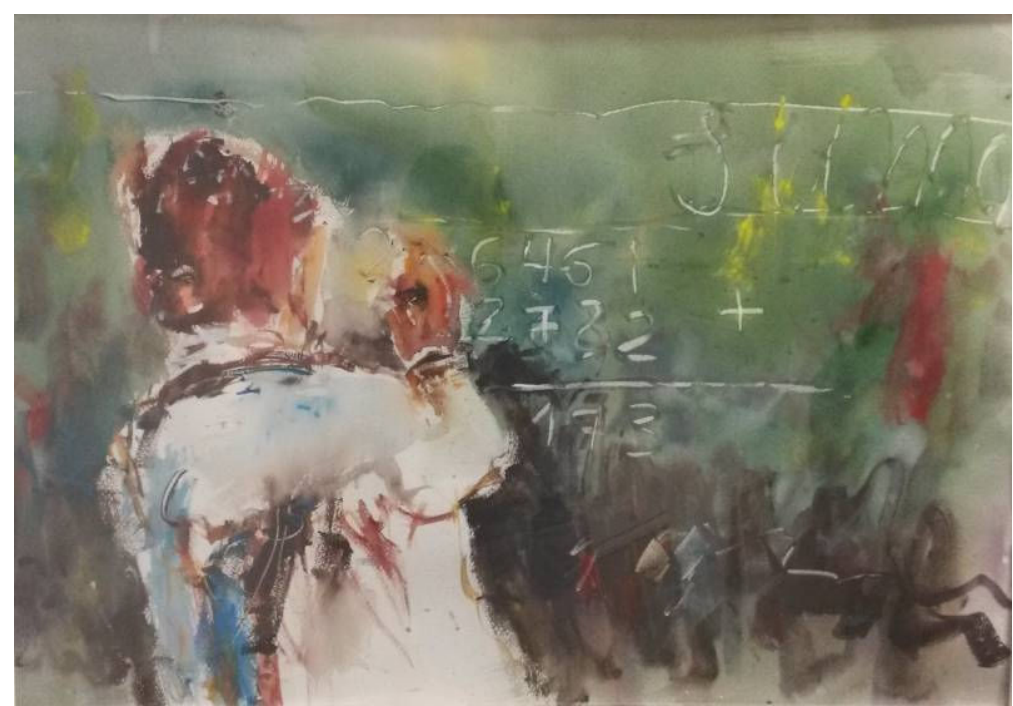

ACCÉSIT

D. Alfredo Sarandeses Lizarazu

"Suma"

(Acuarela sobre papel. 100x70 cm)

El jurado del concurso estuvo compuesto por:

Presidenta: Prof ${ }^{a}$. Dra. Ma José Rebollo Espinosa. Profesora Titular de Universidad. Directora del Museo Pedagógico de la Facultad de CCE de la Universidad de Sevilla. 
Secretario: Prof. Dr. Pablo Álvarez Domínguez. Profesor Acreditado Contratado Doctor. Secretario del Museo Pedagógico de la Facultad de CCE de la Universidad de Sevilla y secretario de la SEPHE.

Vocal 1: Prof. Dr. Luis Méndez Rodríguez. Profesor Titular de Universidad. Director General de Cultura y Patrimonio de la Universidad de Sevilla.

Vocal 2: Prof. Dr. Luis Martínez Montiel. Profesor Titular de Universidad. Director del Secretariado de Patrimonio de la Universidad de Sevilla.

Vocal 3: Prof. Dr. Sergio Villalba Jiménez. Profesor Titular de Universidad. Departamento de Educación Artística. Universidad de Sevilla.

Vocal 4: Prof. Dra. Inmaculada Rodríguez Cunill. Profesora Contratada Doctora. Departamento de Pintura. Universidad de Sevilla.

Invitada con voz, pero sin voto: Prof ${ }^{a}$. $D^{a}$. Marina Núñez Gil. Profesora Titular de Escuela Universitaria. Ex-directora del Museo Pedagógico de la Facultad de CCE de la Universidad de Sevilla.



Luís Martínez, Sergio Villalba, Pablo Álvarez, M José Rebollo, Inmaculada Rodríguez, Marina Núñez y Luís Méndez (de izquierda a derecha). 